\title{
Closure of a full-thickness scalp burn that occurred during hair coloring using a simple skin-stretching method: A case report and review of the literature
}

\author{
SukJoon Oh \\ Department of Burn Reconstructive Surgery, Bestian Seoul Hospital, Seoul, Korea
}

Full-thickness scalp burns secondary to hair coloring are rare; however, such defects can be large and complex reconstruction of hair-bearing tissue may be necessary. Many skin-stretching devices that use gradual traction have been applied to take advantage of the viscoelastic properties of the skin. A 21-year-old female patient was seen with a burn defect on her occipital scalp leading to exposed subcutaneous tissue after chemical application of hair coloring in a salon. The dimensions of the wound were $10 \mathrm{~cm} \times 5 \mathrm{~cm}$, and a skin graft or flap would have been necessary to close the defect. Two long transfixing K-wires $(1.4 \mathrm{~mm})$ and paired 3-wire threads (23 gauge), which are readily available in most hospitals, were applied over a period of 12 days for trichophytic closure of the defect. The remaining scalp scars after primary trichophytic closure with this skin-stretching method were refined with hair follicle transplantation. This skin-stretching method is simple to apply and valuable for helping to close problematic areas of skin shortage that would otherwise require more complicated procedures. This case shows a relatively unknown complication of hair coloring and its treatment.

Keywords Burns, chemical / Scalp defect / Wound healing / Transplantation / Hair follicle
Correspondence: Suk Joon Oh Department of Burn Reconstructive Surgery, Bestian Seoul Hospital, 429 Dogok-ro, Gangnam-gu, Seoul 06208, Korea

Tel: +82-70-7609-9321

Fax: +82-70-7005-4233

E-mail: sjoh46@nate.com

This article was presented at the 14th Korea-Japan Congress of Plastic and Reconstructive Surgery on June 3-5, 2018, in Pyeongchang, Korea.

\section{INTRODUCTION}

Persulfates and hydrogen peroxide are the active ingredients in the highlighting mixture used for hair coloring. Commercially available preparations have a much higher concentration of sulfates (up to 60\%). Off-the scalp preparations generally have a concentration of approximately $25 \%$. Coagulative necrosis results from direct contact of oxidizing salts with tissues. Hydrogen peroxide is also a powerful oxidizing agent. At the concentration of $10 \%$ that is found in many hair coloring materials, it is highly irritating and corrosive, and it can therefore lead to skin blisters and burns $[1,2]$.
The first skin-stretching device (Sure-Closure) was designed by Hirshowitz et al. [3]. Many devices and techniques have been introduced for skin stretching and secure wound closure. However, they were expensive to prepare. The author's technique is a new and simple method to help close problematic areas of skin shortage that would otherwise require more complicated procedures.

\section{CASE}

A 21-year-old female patient was seen with a burn defect on her occipital scalp leading to exposed subcutaneous tissue after 


\section{Fig. 1. Scalp burn wound and skin-stretching closure method}

(A) Full-thickness scalp wound measuring $10 \mathrm{~cm} \times 5 \mathrm{~cm}$ in the occipital area. (B) Two long transfixed K-wires $(1.6 \mathrm{~mm})$ were inserted $2 \mathrm{~cm}$ from the wound margins along the long axis of the wound defect. (C) Paired 3-wire threads (23 gauge) bound with K-wires were inserted at the wound border through the subcutaneous layer. (D) Stretching to approximate the wound edges was started by twisting the paired wire threads on both sides after binding the K-wires along the transverse axis of the wound defect. (E) Complete trichophytic closure was achieved by simple interrupted sutures. (F) This patient experienced partial scar widening 2 years after surgery.
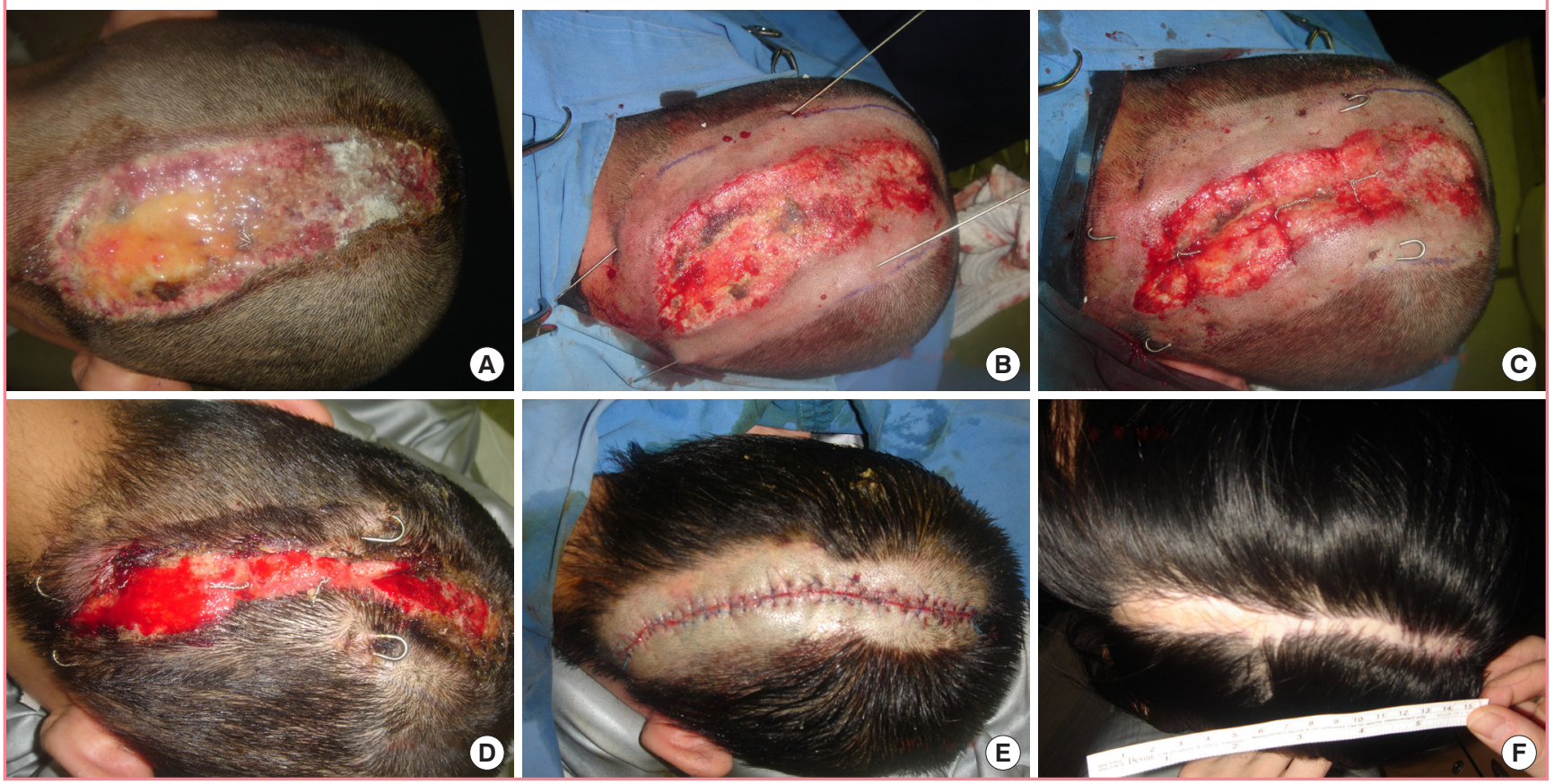

chemical application during hair coloring in a salon. Microbiological examination of a swab obtained from the area of ulceration detected moderately high levels of Pseudomonas aeruginosa. Ciprofloxacin was intravenously injected as an antibiotic to which Pseudomonas aureus is sensitive, and Exsalt T7 (Exciton Technologies Inc., Edmonton, AB, Canada) was used for wound dressing. The dimensions of the wound were $10 \mathrm{~cm} \times 5 \mathrm{~cm}$, and a skin graft or flap would have been required to close the defect (Fig. 1A).

The skin-stretching method was applied, and incremental stretching was performed over a period of 12 days until the defect was closed. This method consists of inserting 2-long transfixing K-wires $(1.6 \mathrm{~mm}) 2 \mathrm{~cm}$ from the wound margins along the long axis of the wound defect and inserting paired 3-wire threads (23 gauge) at the wound border through the subcutaneous layer after binding the $\mathrm{K}$-wires along the transverse axis of the wound defect (Fig. 1B and C). Stretching for approximation of the wound edges was started by twisting the paired wire threads on both sides. The twisting of the paired wire threads was continued until the patient felt discomfort or blanching or shininess was found at the wound edges. This procedure was repeated until wound margins were approximated so they could be sutured together (Fig. 1D). When this skin-stretching device was used, the upper part of the wound was expected to undergo epithelization, but it gradually deteriorated as the hairs were removed and the tissue damage progressed to granulation tissue. Subsequently, complete trichophytic closure was achieved by simple interrupted suturing with 4/0 Vicryl dermal sutures and 4/0 Mersilk skin sutures (Fig. 1E).

The patient experienced partial scar widening 2 years after surgery (Fig. 1F). Therefore, hair follicle transplantation into the scar tissue for aesthetic improvements was performed through the follicular unit extraction method with harvesting from the adjacent occipital scalp (Fig. 2).

\section{DISCUSSION}

Persulfates and hydrogen peroxide are the active ingredients in the highlighting blend used in hair bleaching. Ammonium and potassium persulfate are inorganic salts that are classified as oxidizing agents, in addition to being acidic and flammable. The $\mathrm{pH}$ of the $1 \%$ ammonium persulfate solution is 4 to 6 . Commercially available formulations contain a much higher concentration of sulfates (up to $60 \%$ ) [1].

Off-the scalp preparations generally have a concentration of approximately $25 \%$. Coagulation necrosis is caused by direct 


\section{Fig. 2. Hair follicle transplantation in the scalp scar}

(A) Hair follicle transplantation into the scar tissue for aesthetic improvements was performed by the follicular unit extraction method with harvesting from the adjacent occipital scalp. (B) The donor site of follicular unit extraction and living grafted hairs at 2 months after surgery.

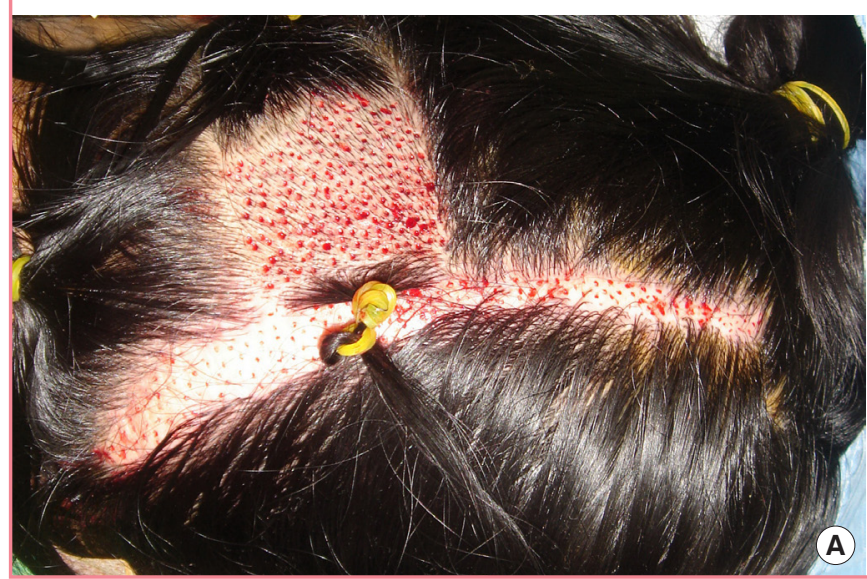

contact between the oxidizing salt and the tissue. Furthermore, strong oxidants also denature proteins, thereby causing cell damage through cytotoxic effects. Hydrogen peroxide is also a powerful oxidizer. It is a clean, colorless, non-flammable liquid and is not well absorbed through intact skin. At the concentrations used for household sterilization purposes $(3 \%-5 \%)$, it is slightly irritating to the skin and mucous membranes. However, at the concentration of $10 \%$ found in many hair-bleaching solutions, it is highly irritating and corrosive, and therefore may cause skin blisters or burns. Prolonged exposure to these oxidizing chemicals causes tissue necrosis and eschar formation, which limits acid penetration [2].

As clinicians, we must educate people about the risk of burns caused by mixtures used for hair coloring, provide information on the safe use of these compounds, and join in current efforts to ban these chemicals from hair coloring products.

The closure of large skin defects is a challenge for surgeons. Large local excisions or acute trauma can create defects that are not amenable to primary closure. In the past, the treatment of large skin defects required the application of a graft or flap.

Due to advances in research into the biomechanical characteristics of skin, pre-suturing techniques [4] and other soft-tissue expansions [5] have been increasingly presented in the literature. These methods manipulate the skin very close to the margins of the wound or within a limited surgical area to facilitate wound closure. Hirshowitz et al. [3] designed the first skinstretching device, among a range of impressive wound closure techniques. This skin-stretching device did not incorporate skin undermining, because undermining together with traction may be detrimental to the viability of the wound margins. The sureclosure device was used to close an 8-cm defect on the lateral side of a patient's leg [6] and escharotomy wounds in a patient

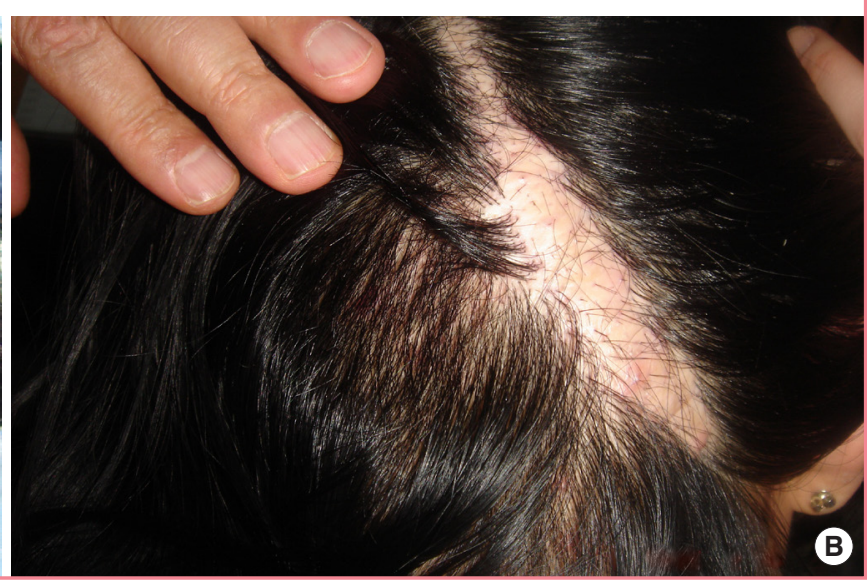

with an acute burn [7].

Other devices and techniques introduced for skin stretching and secure wound closure include Wisebands [8], the TopClosure $3 S$ system [9], the EASApproxi device [10], the shoelace technique [11], and the DermaClose system [12]. Except for the shoelace technique, the devices mentioned are expensive to prepare.

Cases of scalp wound closure using a skin-stretching device can develop marginal alopecia due to reduced blood flow in the sagittal scalp area [13]. Scalp scarring and alopecia can be alleviated with hair follicle transplantation [14].

In addition, 2-long transfixed K-wires $(1.6 \mathrm{~mm})$ and paired 3 -wire threads ( 23 gauge), which are readily available in most hospitals, were utilized to close the large cutaneous scalp wound. This is a valuable and simple method to help close problematic areas of skin shortage that would otherwise require more complicated procedures. Additionally, visible remaining scalp scarring can be refined by hair follicle transplantation using the follicular unit extraction method (Fig. 2).

\section{NOTES}

\section{Conflict of interest}

No potential conflict of interest relevant to this article was reported.

\section{Ethical approval}

The study was approved by the Institutional Review Board of Bestian Seoul Hospital (IRB No. 2018-05-003) and performed in accordance with the principles of the Declaration of Helsinki. Written informed consent was obtained. 


\section{Patient consent}

The patient provided written informed consent for the publication and the use of her images.

\section{ORCID}

SukJoon Oh https://orcid.org/0000-0001-7793-6198

\section{REFERENCES}

1. Pang S, Fiume MZ. Final report on the safety assessment of ammonium, potassium, and sodium persulfate. Int J Toxicol 2001;20 Suppl 3:7-21.

2. Maguina P, Shah-Khan M, An G, et al. Chemical scalp burns after hair highlights. J Burn Care Res 2007;28:361-3.

3. Hirshowitz B, Lindenbaum E, Har-Shai Y. A skin-stretching device for the harnessing of the viscoelastic properties of skin. Plast Reconstr Surg 1993;92:260-70.

4. Liang MD, Briggs P, Heckler FR, et al. Presuturing: a new technique for closing large skin defects: clinical and experimental studies. Plast Reconstr Surg 1988;81:694-702.

5. Manders EK, Schenden MJ, Furrey JA, et al. Soft-tissue expansion: concepts and complications. Plast Reconstr Surg 1984;74:493-507.

6. Ersek RA, Vazquez-Salisbury A. Wound closure using a skin stretching device. Contemp Orthop 1994;28:495-500.

7. Verhaegen PD, Bloemen MC, van der Wal MB, et al. Skin stretching for primary closure of acute burn wounds. Burns 2014;40:1727-37.

8. Barnea Y, Gur E, Amir A, et al. Delayed primary closure of fasciotomy wounds with Wisebands, a skin- and soft tissuestretch device. Injury 2006;37:561-6.

9. Topaz M, Carmel NN, Silberman A, et al. The TopClosure ${ }^{\circledR}$ 3S System, for skin stretching and a secure wound closure. Eur J Plast Surg 2012;35:533-43.

10. Song M, Zhang Z, Liu T, et al. EASApprox $\left({ }^{\circledR}\right)$ skin-stretching system: a secure and effective method to achieve wound closure. Exp Ther Med 2017;14:531-8.

11. Berman SS, Schilling JD, McIntyre KE, et al. Shoelace technique for delayed primary closure of fasciotomies. Am J Surg 1994;167:435-6.

12. Manista GC, Dennis A, Kaminsky M. Surgical management of compartment syndrome and the gradual closure of a fasciotomy wound using a DermaClose device. Trauma Case Rep 2018;14:1-4.

13. Cohen M, Shafir R. Alopecia: an unfavorable result of using a skin-stretching device. Plast Reconstr Surg 1995;96:747-8.

14. Jung S, Oh SJ, Koh SH. Hair follicle transplantation on scar tissue. J Craniofac Surg 2013;24:1239-41. 